\title{
Alicyclobacillus aeris sp. nov., a novel ferrous- and sulfur-oxidizing bacterium isolated from a copper mine
}

\author{
Xu Guo, Xiao-Yan You, Li-Jun Liu, Jia-Yue Zhang, Shuang-Jiang Liu \\ and Cheng-Ying Jiang
}

Correspondence

Cheng-Ying Jiang

jiangcy@sun.im.ac.cn

\author{
State Key Laboratory of Microbial Resources, Institute of Microbiology, Chinese Academy of \\ Sciences, Beijing 100101, PR China
}

A novel mesophilic, acidophilic, endospore-forming bacterium, designated strain $\mathrm{ZJ}-6^{\top}$, was isolated from $\mathrm{Zi}$-Jin copper mine in Inner Mongolia, China. Cells of strain $\mathrm{ZJ}-6^{\top}$ were rod-shaped, stained Gram-positive or were Gram-variable, and grew aerobically at $25-35{ }^{\circ} \mathrm{C}$ (optimum, $30{ }^{\circ} \mathrm{C}$ ) and $\mathrm{pH}$ 2.0-6.0 (optimum, $\mathrm{pH}$ 3.5). 16S rRNA gene sequence analysis showed that strain $\mathrm{ZJ}-6^{\top}$ was related phylogenetically to members of the genus Alicyclobacillus, with $16 \mathrm{~S}$ rRNA gene sequence similarities of 89.5-94.2\%. Cells contained MK-7 as the major quinone and the DNA G $+C$ content was 51.2 mol\%. Strain $Z \mathrm{~J}-6^{\top}$ possessed a number of phenotypic characteristics that differentiated it from recognized Alicyclobacillus species, including its growth temperature, assimilation of various carbon sources, production of acids from a range of compounds, and the ability to grow chemoautotrophically using ferrous iron, elemental sulfur and tetrathionate as electron donors. The predominant cellular fatty acids of strain $\mathrm{ZJ}-6^{\top}$ were anteiso$\mathrm{C}_{15: 0}(67.1 \%)$, iso- $\mathrm{C}_{16: 0}(7.7 \%)$ and anteiso- $\mathrm{C}_{17: 0}(7.4 \%) ; \omega$-alicyclic fatty acids were not found. On the basis of these results, it is concluded that strain $\mathrm{ZJ}-6^{\top}$ represents a novel species within the genus Alicyclobacillus, for which the name Alicyclobacillus aeris sp. nov. is proposed; the type strain is $\mathrm{ZJ}-6^{\top}$ (=CGMCC $1.7072^{\top}=$ NBRC $\left.104953^{\top}\right)$.
Members of the genus Alicyclobacillus stain Gram-positive or are Gram-variable and are strictly aerobic, thermophilic or moderately thermophilic, acidophilic, spore-forming rods. Many members of the genus formerly belonged to the genus Bacillus, but were reclassified as members of a new genus, Alicyclobacillus (Wisotzkey et al., 1992), because of their distinct 16S rRNA gene sequences and the occurrence of unique $\omega$-alicyclic fatty acids. However, the description of the genus was subsequently amended to include organisms that possess straight- and branched-chain fatty acids due to the discovery of some species that do not possess $\omega$-alicyclic fatty acids, e.g. Alicyclobacillus pomorum (Goto et al., 2003), Alicyclobacillus macrosporangiidus and Alicyclobacillus contaminans (Goto et al., 2007), Alicyclobacillus pohliae (Imperio et al., 2008) and Alicyclobacillus ferrooxydans (Jiang et al., 2008). At the time of writing (June 2008), the genus Alicyclobacillus was composed of 21 species with validly published names, two

The GenBank/EMBL/DDBJ accession number for the 16S rRNA gene sequence of strain $\mathrm{ZJ}-6^{\top}$ is FM179383.

Phylogenetic trees based on 16S rRNA gene sequences constructed using the minimum-evolution and maximum-parsimony methods and a table showing the cellular fatty acid compositions of strain $\mathrm{ZJ}-6^{\top}$ and related strains are available as supplementary material with the online version of this paper. subspecies and two genomic species (Fig. 1), with Alicyclobacillus acidocaldarius as the type species (Wisotzkey et al., 1992; Goto et al., 2006). Although most members of the genus Alicyclobacillus are heterotrophic organisms that often inhabit fruit beverages, acidic geothermal environments such as geothermal water (Hiraishi et al., 1997; Nicolaus et al., 1998) and soil (Hippchen et al., 1981; Tsuruoka et al., 2003), Alicyclobacillus tolerans, Alicyclobacillus disulfidooxidans and $A$. ferrooxydans are able to oxidize ferrous iron, elemental sulfur and sulfides for growth (Kovalenko \& Malakhova, 1983; Dufresne et al., 1996, Jiang et al., 2008). Recently, some Alicyclobacillus-like strains that were also capable of oxidizing ferrous iron and metal sulfides were isolated from metal- or metal sulfide-rich ores and from a bioleaching column (Johnson \& Hallberg, 2007; Wakeman, et al., 2008).

During the course of a microbiological consortium survey of solfataric mine drainage (temperature and $\mathrm{pH}$ ranges were $25-35{ }^{\circ} \mathrm{C}$ and $2.5-5.5$, respectively) of $\mathrm{Zi}$-Jin copper mine located in Inner Mongolia, China, an acidophilic bacterial strain, strain $\mathrm{ZJ}-6^{\mathrm{T}}$, was isolated and purified. Strain ZJ-6 $6^{\mathrm{T}}$ grew heterotrophically as well as chemolithotrophically. It oxidized ferrous iron, elemental sulfur and tetrathionate and obtained energy for growth. Results of a 


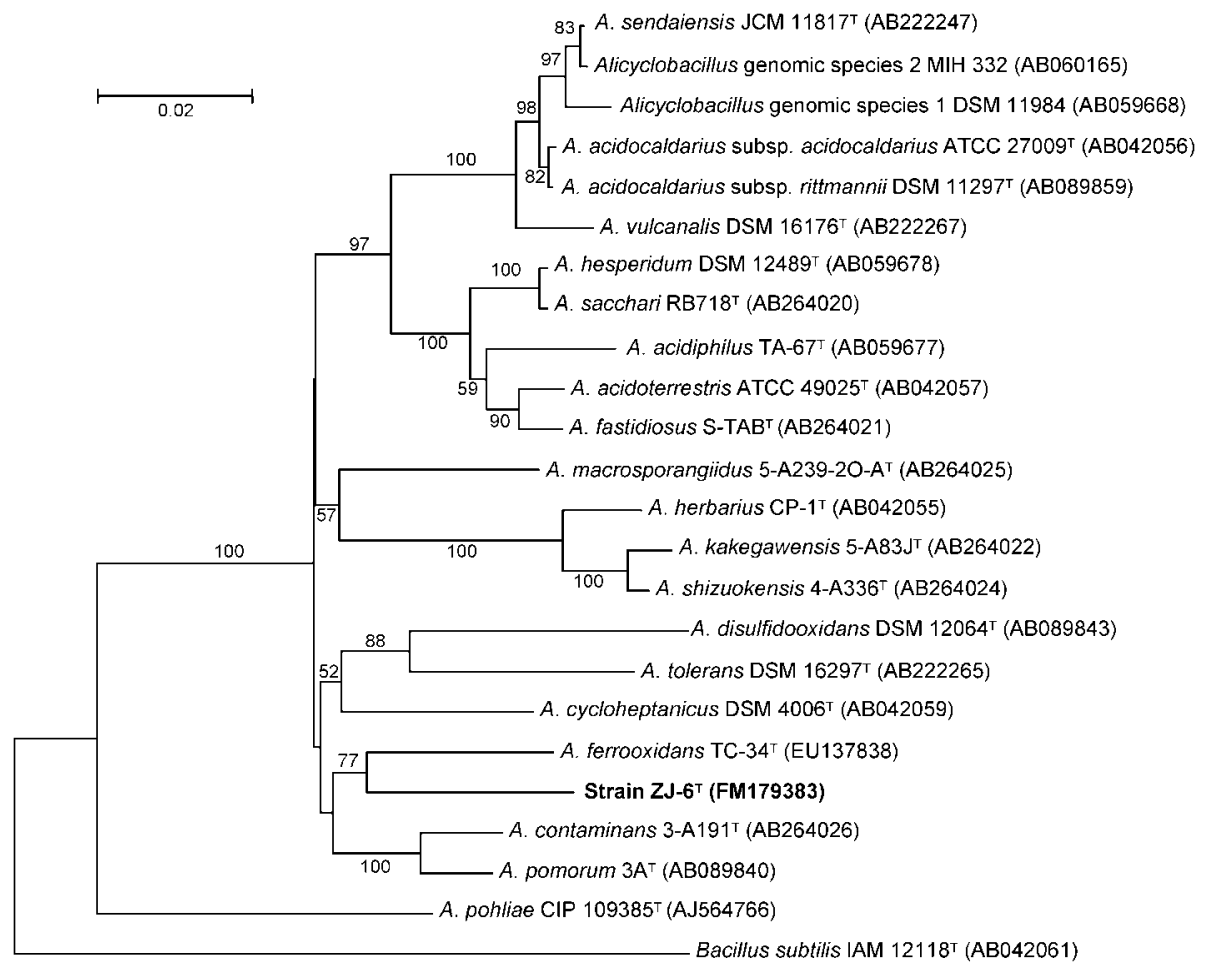

Fig. 1. Phylogenetic tree constructed with the neighbour-joining method based on $16 \mathrm{~S}$ rRNA gene sequence evolutionary distances among strain $\mathrm{ZJ}-6^{\top}$ and other Alicyclobacillus species. B. subtilis IAM $12118^{\top}$ was used as the outgroup. GenBank accession numbers are given in parentheses. Numbers at nodes represent confidence levels from 1000 replicate bootstrap samplings; only values greater than $50 \%$ are shown. Bar, 0.02 substitutions per nucleotide position.

polyphasic taxonomic study of strain $\mathrm{ZJ}-6^{\mathrm{T}}$ involving assessment of its physiological, chemotaxonomic and phylogenetic properties are reported here.

Strain $\mathrm{ZJ}-6^{\mathrm{T}}$ was isolated from solfataric copper mine drainage using modified Norris broth (Norris et al., 1996; Jiang et al., 2008). The collected samples were first enriched at $30{ }^{\circ} \mathrm{C}$ with the modified broth. After enriching three times, the enriched culture was serially diluted (10-fold) in tubes containing $0.9 \mathrm{ml}$ modified Norris broth. Dilutions of $0.2 \mathrm{ml}$ were spread onto plates to cultivate separate colonies. Further purification of single colonies was carried out by repeated streaking on modified Norris plates. The plates were incubated at $30{ }^{\circ} \mathrm{C}$ for 7 days. The purity of the strain was checked by $16 \mathrm{~S}$ rRNA gene sequence analysis and the phenotypic homogeneity of culture.

Cell morphology and flagellation were examined during the exponential growth phase by scanning electron microscopy (Quanta 200; FEI) and transmission electron microscopy (H600; Hitachi). Physiological and chemotaxonomic characterization of strain ZJ- $6^{\mathrm{T}}$ was carried out with Bacillus acidocaldarius medium (BAM medium; Deinhard et al., 1987a). Gram-staining reactions were determined according to the method described by Gerhardt et al. (1994). Endospore formation was observed after malachite green staining of cells from 6-day-old cultures in BAM broth. Unless otherwise stated, catalase and oxidase activities, the Voges-Proskauer reaction, carbon source utilization tests and other biochemical characterization tests were performed as described previously (Deinhard et al., 1987a, b; Albuquerque et al., 2000; Goto et al., 2002; Jiang et al., 2008) in BAM media. Biochemical characterization was carried out according to Bergey's Manual of Systematic Bacteriology (Claus \& Berkeley, 1986) and the Determinative Manual for Routine Bacteriology (Dong \& Cai, 2001), complemented with the API ZYM, API 20NE and API $50 \mathrm{CH}$ systems (all bioMérieux) using BAM basal salts medium. Cell concentrates were resuspended in $15 \mathrm{ml}$ modified BAM medium ( $\mathrm{pH} 4.0$ ) with $0.03 \mathrm{~g}$ bromophenol blue $\mathrm{l}^{-1}$ as indicator and samples were distributed in the API $50 \mathrm{CH}$ test strip wells to test for acid production. The growth temperature range was examined with a TN3F temperature-gradient incubator (Advantec). Growth at various $\mathrm{pH}(0.5-7.0$ at intervals of 0.5 , adjusted by the addition of $1 \mathrm{M} \mathrm{H}_{2} \mathrm{SO}_{4}$ ) was examined in BAM medium. Cell growth was determined by measuring the increase in $\mathrm{OD}_{578}$. The following compounds were tested as electron donors: elemental sulfur $\left(5.0 \mathrm{~g} \mathrm{l}^{-1}\right.$, sterilized by heating at $100{ }^{\circ} \mathrm{C}$ for $1 \mathrm{~h}$, repeated twice on consecutive days); $\mathrm{K}_{2} \mathrm{~S}_{4} \mathrm{O}_{6}\left(10 \mathrm{mmol} \mathrm{l}^{-1}\right) ; \mathrm{Na}_{2} \mathrm{~S}_{2} \mathrm{O}_{3}\left(5 \mathrm{mmol} \mathrm{l}^{-1}\right) ; \mathrm{FeSO}_{4}$ $\left(14.0 \mathrm{~g} \mathrm{l}^{-1}\right)$; and $\mathrm{FeS}_{2}\left(5.0 \mathrm{~g} \mathrm{l}^{-1}\right)$. Growth under anaerobic 
conditions was examined according to Dong \& Cai (2001) in both modified Norris broth and modified liquid BAM medium.

For analysis of cellular fatty acid patterns, strain ZJ-6 ${ }^{\mathrm{T}}$ was grown for 5 days at $30{ }^{\circ} \mathrm{C}$ on BAM agar. Cells were harvested from the plates and fatty acids were methylated and analysed using the Sherlock Microbial Identification system (MIDI), which is based on GC, according to the manufacturer's instructions (Microbial ID). Isoprenoid quinones were extracted from freeze-dried cells $(200 \mathrm{mg})$ with chloroform/methanol $(2: 1, \mathrm{v} / \mathrm{v})$ and then separated from other components by TLC. The purified isoprenoid quinones were analysed by reversed-phase HPLC equipped with a Zorbax ODS $\mathrm{C}_{18}$ column (Agilent) and using acetonitrile/isopropylalcohol $(2: 1.2, \mathrm{v} / \mathrm{v})$ as the mobile phase. The DNA G+C content was determined by the thermal denaturation method (Marmur \& Doty, 1962) using DNA from Escherichia coli K-12 as a reference.

The nearly complete $16 \mathrm{~S}$ rRNA gene of strain $\mathrm{ZJ}-6^{\mathrm{T}}$ was amplified with the primers 27f (5'-AGAGTTTGATCTTGGCTCAG-3') and 1492r (5'-GGTTACCTTGTTACGACTT- $3^{\prime}$ ) and sequenced. Alignments of $16 \mathrm{~S}$ rRNA gene sequences were performed with the program CLUSTAL_X version 1.64b (Thompson et al., 1997) and positions with insertions or deletions were excluded during calculations. Phylogenetic trees were constructed with the neighbourjoining, maximum-parsimony and minimum-evolution tree-making algorithms using MEGA 3.1 (Saitou \& Nei, 1987; Kumar et al., 2004) based on evolutionary distances that were calculated with the Kimura two-parameter model (Kimura, 1980).

Cells of strain ZJ-6 ${ }^{\mathrm{T}}$ stained Gram-positive or were Gramvariable and were strictly aerobic, endospore-forming, straight rods that possessed peritrichous flagella (Fig. 2). Cells were negative for catalase and oxidase. Colonies of strain ZJ-6 ${ }^{\mathrm{T}}$ on modified Norris plates were brown-centred with yellow-orange peripheries, circular, flat and entire with diameters of $0.3-0.5 \mathrm{~mm}$ after incubation for 56 days. Colonies on BAM plates were creamy white, circular and entire with diameters of $0.5-1.0 \mathrm{~mm}$ after incubation for 3-5 days. Carbon compounds that were tested for their ability to support growth and produce acid are given in Table 1 and the species description.

Strain ZJ $-6^{\mathrm{T}}$ was mesophilic and grew at $25-35^{\circ} \mathrm{C}$ with optimal growth at $30{ }^{\circ} \mathrm{C}$, thus differing from the known thermophilic species of the genus Alicyclobacillus, which grow optimally at $42-60{ }^{\circ} \mathrm{C}$ and do not grow below $25{ }^{\circ} \mathrm{C}$ (Wisotzkey et al., 1992; Goto et al., 2003) (Table 1). Strain $\mathrm{ZJ}-6^{\mathrm{T}}$ grew at $\mathrm{pH} 2.0-6.0$, with optimal growth at $\mathrm{pH} 3.5$. Strain ZJ $-6^{\mathrm{T}}$ oxidized ferrous iron, elemental sulfur and $\mathrm{K}_{2} \mathrm{~S}_{4} \mathrm{O}_{6}$ as electron donors for growth; however, no growth was observed using $\mathrm{FeS}_{2}$ as the electron donor. Anaerobic growth did not occur. Supplementing Norris broth (mineral salts medium; Norris et al., 1996) containing ferrous iron, elemental sulfur or $\mathrm{K}_{2} \mathrm{~S}_{4} \mathrm{O}_{6}$ as electron donor with yeast extract stimulated growth of strain ZJ-6 ${ }^{\mathrm{T}}$.

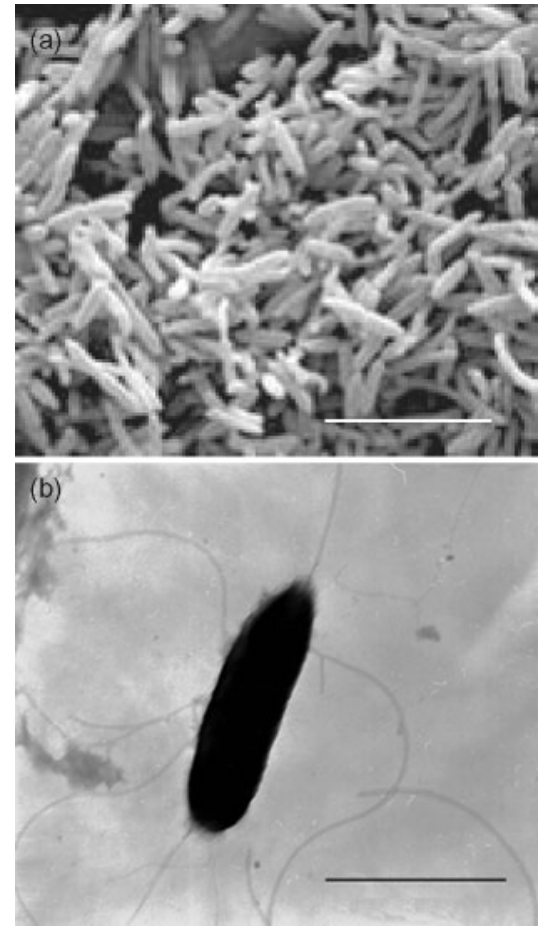

Fig. 2. Scanning (a; bar, $5 \mu \mathrm{m})$ and transmission (b; bar, $1 \mu \mathrm{m})$ electron micrographs showing the morphology of strain $\mathrm{ZJ}-6^{\top}$ and peritrichous flagella.

The predominant cellular fatty acids of strain $\mathrm{ZJ}-6^{\mathrm{T}}$ were anteiso- $\mathrm{C}_{15: 0}(67.1 \%)$, iso- $\mathrm{C}_{16: 0}(7.7 \%)$, anteiso- $\mathrm{C}_{17: 0}$ $(7.4 \%)$, iso- $\mathrm{C}_{14: 0}(5.1 \%)$ and iso- $\mathrm{C}_{15: 0}(3.7 \%) . \omega$-Alicyclic fatty acid was not detected; this was also the case in $A$. pomorum, A. macrosporangiidus, A. contaminans, A. pohliae and A. ferrooxydans, but not in the other Alicyclobacillus species, which contain $\omega$-alicyclic fatty acid as a characteristic fatty acid. The fatty acid profiles of strain ZJ- $6^{\mathrm{T}}$ and other Alicyclobacillus strains are provided in Supplementary Table S1 (available in IJSEM Online). Strain ZJ- $6^{\mathrm{T}}$ had MK-7 as the major respiratory quinone, which accounted for $97 \%$ of the total, and MK-6 as a minor component (3\%). The DNA $\mathrm{G}+\mathrm{C}$ content of strain ZJ-6 ${ }^{\mathrm{T}}$ was $51.2 \mathrm{~mol} \%$, which is within the range for recognized Alicyclobacillus species (48.7$62.7 \mathrm{~mol} \%$ ) (Karavaiko et al., 2005).

16S rRNA gene sequence analysis showed that strain ZJ-6 ${ }^{\mathrm{T}}$ was related phylogenetically to members of the genus Alicyclobacillus (similarities of 89.5-94.2\% to type strains of species with validly published names), with the highest similarity $(94.2 \%)$ to the type strain of A. ferrooxydans. The neighbour-joining tree (Fig. 1) showed that strain ZJ-6 ${ }^{\mathrm{T}}$ clustered with strains of Alicyclobacillus species. This cluster was also supported topologically by the minimum-evolution and maximum-parsimony trees (see Supplementary Fig. S1 available in IJSEM Online).

Strain ZJ $-6^{\mathrm{T}}$ showed a range of phenotypic characteristics that differ from those of previously described 
Table 1. Differential phenotypic characteristics of strain $\mathrm{ZJ}-6^{\top}$ and strains of other Alicyclobacillus species

Strains: 1, ZJ-6 ${ }^{\mathrm{T}}$ (A. aeris sp. nov.); 2, A. ferrooxydans TC-34 ${ }^{\mathrm{T}} ; 3$, A. pomorum $3 \mathrm{~A}^{\mathrm{T}}$; 4, A. contaminans 3-A191 ${ }^{\mathrm{T}}$; 5, A. tolerans $\mathrm{K1}^{\mathrm{T}}$; 6, A. hesperidum DSM $12489^{\mathrm{T}} ; 7$, A. macrosporangiidus DSM $17980^{\mathrm{T}} ; 8$, A. acidoterrestris DSM $3922^{\mathrm{T}} ; 9$, A. sacchari DSM $17974^{\mathrm{T}} ; 10$, A. cycloheptanicus DSM 4006 ${ }^{\mathrm{T}}$; 11, A. acidiphilus TA $-67^{\mathrm{T}} ; 12$, A. fastidiosus DSM $17978^{\mathrm{T}} ; 13$, A. kakegawensis DSM 17979 $; 14$, A. shizuokensis DSM 17981 ${ }^{\mathrm{T}}$; 15, A. herbarius DSM $13609^{\mathrm{T}} ; 16$, A. disulfidooxidans DSM $12064^{\mathrm{T}} ; 17$, A. vulcanalis $\mathrm{CsHg} 2^{\mathrm{T}} ; 18$, A. sendaiensis $\mathrm{JCM} 11817^{\mathrm{T}} ; 19$, A. pohliae MP4 ${ }^{\mathrm{T}}$; 20, A. acidocaldarius subsp. acidocaldarius DSM $446^{\mathrm{T}}$. For all tests, A. cycloheptanicus DSM $4006^{\mathrm{T}}$ was run in parallel with strain ZJ-6 ${ }^{\mathrm{T}}$. When data for A. cycloheptanicus DSM $4006^{\mathrm{T}}$ obtained in this study differed from that reported previously (Albuquerque et al., 2000; Goto et al., 2007), the results from this study are given and the previous results are provided in parentheses. Symbols: +, positive; -, negative; ND, not determined; w, weakly positive; v, variable between strains.

\begin{tabular}{|c|c|c|c|c|c|c|c|c|c|c|c|c|c|c|c|c|c|c|c|c|}
\hline Characteristic & $1^{\star}$ & $2^{*}$ & $3 \dagger$ & $4 \dagger$ & $5 \ddagger$ & $6 \ddagger$ & $7 \dagger$ & $8 \ddagger$ & $9 \dagger$ & $10^{\star} \ddagger$ & $11 \ddagger$ & $12 \dagger$ & $13 \dagger$ & $14 \dagger$ & $15 \ddagger$ & $16 \ddagger$ & $17 \S$ & $18 \|$ & 199 & $20 \ddagger$ \\
\hline $\begin{array}{l}\text { Growth temperature } \\
\left({ }^{\circ} \mathrm{C}\right)\end{array}$ & $\begin{array}{r}25- \\
35\end{array}$ & $\begin{array}{r}17- \\
40\end{array}$ & $\begin{array}{r}30- \\
60\end{array}$ & $\begin{array}{r}35- \\
60\end{array}$ & $\begin{array}{r}20- \\
55\end{array}$ & $\begin{array}{r}35- \\
60\end{array}$ & $\begin{array}{r}35- \\
60\end{array}$ & $\begin{array}{r}35- \\
55\end{array}$ & $\begin{array}{r}30- \\
55\end{array}$ & $\begin{array}{r}40- \\
53\end{array}$ & $\begin{array}{r}20- \\
50\end{array}$ & $\begin{array}{r}20- \\
55\end{array}$ & $\begin{array}{r}40- \\
60\end{array}$ & $\begin{array}{r}35- \\
60\end{array}$ & $\begin{array}{r}35- \\
65\end{array}$ & $\begin{array}{c}4- \\
40\end{array}$ & $\begin{array}{r}35- \\
65\end{array}$ & $\begin{array}{r}40- \\
65\end{array}$ & $\begin{array}{r}42- \\
60\end{array}$ & $\begin{array}{r}45- \\
70\end{array}$ \\
\hline Growth $\mathrm{pH}$ range & $\begin{array}{r}2.0- \\
6.0\end{array}$ & $\begin{array}{r}2.0- \\
6.0\end{array}$ & $\begin{array}{r}3.0- \\
6.0\end{array}$ & $\begin{array}{r}3.0- \\
6.0\end{array}$ & $\begin{array}{r}1.5- \\
5.0\end{array}$ & $\begin{array}{r}2.0- \\
6.0\end{array}$ & $\begin{array}{r}3.0- \\
6.5\end{array}$ & $\begin{array}{r}2.2- \\
5.8\end{array}$ & $\begin{array}{r}2.0- \\
6.5\end{array}$ & $\begin{array}{r}3.0- \\
5.5\end{array}$ & $\begin{array}{r}2.5- \\
5.5\end{array}$ & $\begin{array}{r}2.0- \\
5.5\end{array}$ & $\begin{array}{r}3.0- \\
6.5\end{array}$ & $\begin{array}{r}3.0- \\
6.5\end{array}$ & $\begin{array}{r}3.0- \\
6.5\end{array}$ & $\begin{array}{r}0.5- \\
6.0\end{array}$ & $\begin{array}{r}2.0- \\
6.0\end{array}$ & $\begin{array}{r}2.5- \\
6.5\end{array}$ & $\begin{array}{r}4.5- \\
7.5\end{array}$ & $\begin{array}{r}2.0- \\
6.0\end{array}$ \\
\hline $\begin{array}{l}\text { DNA G + C content } \\
(\mathrm{mol} \%)\end{array}$ & 51.2 & 48.6 & 53.1 & 60.3 & $\begin{array}{c}48.7 \pm \\
0.6\end{array}$ & 53.3 & 62.5 & 52.2 & 56.6 & 55.6 & 54.1 & 53.9 & $\begin{array}{c}61.5 \pm \\
0.2\end{array}$ & 60.5 & 56.2 & 53.0 & 62.0 & 62.3 & 55.1 & 60.3 \\
\hline Oxidase & - & + & + & - & w & - & - & - & - & + & - & - & - & - & - & - & $\mathrm{ND}$ & - & - & - \\
\hline Catalase & - & + & + & - & $\mathrm{w}$ & - & $\mathrm{w}$ & + & - & + & + & + & $\mathrm{w}$ & + & + & - & - & - & - & + \\
\hline \multicolumn{21}{|l|}{ Hydrolysis of: } \\
\hline Gelatin & + & - & + & + & - & + & - & + & + & - & - & + & - & - & - & - & $\mathrm{ND}$ & $\mathrm{ND}$ & $\mathrm{ND}$ & + \\
\hline Starch & - & + & + & - & + & + & - & - & + & - & - & - & - & - & - & + & + & $\mathrm{ND}$ & $\mathrm{ND}$ & + \\
\hline $\begin{array}{l}\text { Nitrate reduction to } \\
\text { nitrite }\end{array}$ & + & - & - & - & - & - & - & - & - & - & - & - & - & - & + & - & ND & + & $\mathrm{ND}$ & - \\
\hline Growth in $5 \% \mathrm{NaCl}$ & - & - & - & - & ND & - & + & + & - & + & - & - & - & + & + & $\mathrm{ND}$ & - & $\mathrm{ND}$ & - & - \\
\hline \multicolumn{21}{|l|}{ Acid production from: } \\
\hline Glycerol & + & - & + & + & $\mathrm{W}$ & + & + & + & + & - & - & - & - & - & + & + & ND & + & - & + \\
\hline D-Arabinose & - & - & - & - & $\mathrm{W}$ & - & + & - & - & + & + & + & + & - & + & + & ND & + & + & - \\
\hline L-Arabinose & + & + & - & + & $\mathrm{w}$ & + & + & + & + & + & + & + & + & + & + & + & $\mathrm{ND}$ & + & + & + \\
\hline D-Xylose & + & - & - & + & + & + & + & - & + & + & + & + & + & + & + & + & ND & $\mathrm{ND}$ & + & + \\
\hline L-Xylose & + & - & - & - & + & - & + & - & - & + & - & - & + & + & - & + & ND & $\mathrm{ND}$ & + & - \\
\hline Methyl $\beta$-xyloside & - & + & - & - & + & - & - & - & + & - & - & + & - & - & - & $\mathrm{ND}$ & - & $\mathrm{ND}$ & - & - \\
\hline D-Galactose & + & - & - & + & + & + & + & + & + & - & + & + & + & + & + & - & $\mathrm{ND}$ & + & + & + \\
\hline L-Sorbose & - & - & + & $\mathrm{v}$ & + & - & + & - & - & + & + & - & + & - & - & + & $\mathrm{ND}$ & $\mathrm{ND}$ & + & - \\
\hline Rhamnose & + & - & - & $\mathrm{v}$ & - & - & + & + & + & + & - & + & + & - & + & - & - & - & + & + \\
\hline Mannitol & + & - & + & + & + & + & - & + & + & + & - & + & + & + & + & + & + & ND & - & + \\
\hline Sorbitol & + & + & - & - & - & - & + & + & - & + & + & - & + & - & - & - & - & - & - & - \\
\hline $\begin{array}{l}\text { Methyl } \alpha \text {-D- } \\
\text { mannoside }\end{array}$ & - & + & - & - & + & - & + & - & - & - & - & - & + & - & + & $\mathrm{ND}$ & ND & $\mathrm{ND}$ & - & - \\
\hline Amygdalin & - & - & + & - & + & - & - & - & - & - & - & - & + & - & + & + & ND & - & - & - \\
\hline Arbutin & - & - & - & + & - & + & - & - & + & - & + & - & + & + & + & - & $\mathrm{w}$ & + & $\mathrm{w}$ & + \\
\hline Aesculin & + & + & + & + & + & + & + & - & - & + & + & - & + & + & + & + & $\mathrm{ND}$ & - & + & + \\
\hline Salicin & - & - & + & $\mathrm{v}$ & - & - & + & - & + & - & + & - & + & + & + & - & - & + & $\mathrm{w}$ & + \\
\hline Cellobiose & - & - & - & + & - & + & - & + & + & - & + & - & + & + & + & + & ND & + & + & + \\
\hline Maltose & - & - & + & + & w & + & + & + & + & - & + & - & + & + & + & + & ND & + & + & + \\
\hline Lactose & - & - & - & $\mathrm{v}$ & $\mathrm{w}$ & - & + & + & + & - & + & - & + & - & + & - & - & + & + & + \\
\hline Melibiose & - & - & - & - & - & - & - & $\mathrm{W}$ & + & - & - & + & - & + & + & + & + & - & + & + \\
\hline Sucrose & - & - & + & + & + & + & + & + & + & - & + & - & + & + & + & + & + & + & + & + \\
\hline Trehalose & - & + & + & + & + & + & + & + & + & $-(+)$ & + & + & + & + & + & - & + & + & $\mathrm{w}$ & + \\
\hline$\beta$-Gentiobiose & - & - & - & $\mathrm{v}$ & $\mathrm{ND}$ & w & + & - & + & - & + & - & + & + & + & $\mathrm{ND}$ & - & $\mathrm{ND}$ & + & - \\
\hline Turanose & - & + & + & - & $\mathrm{w}$ & + & - & - & + & - & + & - & + & - & + & - & + & + & + & - \\
\hline D-Lyxose & - & + & - & - & $\mathrm{ND}$ & - & + & - & - & + & - & + & + & - & - & $\mathrm{ND}$ & $\mathrm{ND}$ & $\mathrm{ND}$ & + & - \\
\hline D-Tagatose & - & + & + & $\mathrm{v}$ & $\mathrm{w}$ & - & - & - & - & + & - & + & $\mathrm{V}$ & - & - & + & $\mathrm{ND}$ & $\mathrm{ND}$ & + & - \\
\hline 5-Ketogluconate & + & + & + & - & + & + & - & - & - & + & - & - & - & - & + & + & $\mathrm{w}$ & - & + & - \\
\hline
\end{tabular}

${ }^{*}$ Data from this study.

$\dagger$ Data from Goto et al. (2007).

¥Data from Karavaiko et al. (2005).

$\$$ Data from Simbahan et al. (2004).

IIData from Tsuruoka et al. (2003).

SData from Imperio et al. (2008). 
Alicyclobacillus species. These included differences in the ability to assimilate various carbon sources, produce acids, reduce nitrate, and oxidize ferrous iron and inorganic sulfuric compounds (Table 1). The major cellular fatty acids of strain $\mathrm{ZJ}-6^{\mathrm{T}}$ were also significantly different from those of other Alicyclobacillus species.

Based on the above phenotypic and phylogenetic studies, it is clear that strain ZJ-6 ${ }^{\mathrm{T}}$ represents a novel species of the genus Alicyclobacillus, for which the name Alicyclobacillus aeris sp. nov. is proposed.

\section{Description of Alicyclobacillus aeris sp. nov.}

Alicyclobacillus aeris (ae' ris. L. gen. neut. n. aeris of ore/ copper).

Cells are rods with rounded ends $(0.4-0.6 \times 1.5-2.5 \mu \mathrm{m})$, stain Gram-positive or are Gram-variable, and are strictly aerobic, motile and endospore-forming. Colonies on modified Norris broth are brown-centred with yelloworange peripheries, circular, flat and entire with diameters of $0.3-0.5 \mathrm{~mm}$ after incubation for 5-6 days. Colonies on BAM plates are creamy white, circular and entire with diameters of $0.5-1.0 \mathrm{~mm}$ after incubation for 3-5 days. Temperature range for growth is $25-35{ }^{\circ} \mathrm{C}$; optimum growth temperature is $30^{\circ} \mathrm{C}$. Optimum $\mathrm{pH}$ for growth is 3.5; growth occurs at $\mathrm{pH} 2.0-6.0$. Cells grow well in BAM medium containing $0-2 \%(\mathrm{w} / \mathrm{v}) \mathrm{NaCl}$, but are inhibited by $3 \% \mathrm{NaCl}$. Oxidase, catalase and Voges-Proskauer reactions are negative. Indole and $\mathrm{H}_{2} \mathrm{~S}$ are not produced. Reduces nitrate. Gelatin and aesculin are hydrolysed, but casein, starch and Tween 80 are not. Leucine arylamidase, valine arylamidase, cystine arylamidase, trypsin, chymotrypsin, acid phosphatase, naphthol-AS-BI-phosphohydrolase, $\alpha$-glucosidase and $\beta$-glucosidase are positive; alkaline phosphatase, esterase (C4), esterase lipase (C8), lipase (C14), $\alpha$-galactosidase, $\beta$-galactosidase, $\beta$-glucuronidase, $N$-acetyl- $\beta$-glucosaminidase, $\alpha$-mannosidase and $\alpha$-fucosidase are absent. Grows on the carbon sources peptone, starch, raffinose, D-galactose, D-glucose, L-arabinose and adipate, but not on D-glucitol, L-rhamnose, D-sorbose, Dmannose, maltose, sucrose, D-fructose, D-xylose, cellobiose, inulin, mannitol, phenylacetate or caprate. Acid is produced from glycerol, erythritol, L-arabinose, ribose, Dxylose, L-xylose, adonitol, galactose, D-glucose, D-fructose, D-mannose, mannitol, sorbitol, aesculin, xylitol, fucose, arabitol and 5-ketogluconate, but not from D-arabinose, methyl $\beta$-D-xyloside, L-sorbose, rhamnose, dulcitol, inositol, methyl $\alpha$-D-mannoside, methyl $\alpha$-D-glucoside, $N$ acetylglucosamine, amygdalin, arbutin, salicin, cellobiose, maltose, lactose, melibiose, sucrose, trehalose, inulin, melezitose, raffinose, starch, glycogen, $\beta$-gentiobiose, turanose, D-lyxose, D-tagatose, gluconate or 2-ketogluconate. Can grow chemoautotrophically by using ferrous iron, elemental sulfur and $\mathrm{K}_{2} \mathrm{~S}_{4} \mathrm{O}_{6}$ as electron donors. Major fatty acids are anteiso- $\mathrm{C}_{15: 0}$, iso- $\mathrm{C}_{16: 0}$, anteiso$\mathrm{C}_{17: 0}$, iso- $\mathrm{C}_{14: 0}$ and iso- $\mathrm{C}_{15: 0}$. The major isoprenoid quinone is MK-7.
The type strain is $\mathrm{ZJ}-6^{\mathrm{T}} \quad\left(=\mathrm{CGMCC} 1.7072^{\mathrm{T}}=\mathrm{NBRC}\right.$ $\left.104953^{\mathrm{T}}\right)$, isolated from $\mathrm{Zi}$-Jin copper mine, Inner Mongolia, China. The DNA G $+\mathrm{C}$ content of the type strain is $51.2 \mathrm{~mol} \%$.

\section{Acknowledgements}

We thank Professor Trüper for his valuable advice on nomenclature. This work was supported by grants (30670018 and 30870039) from the National Nature Science Foundation of China.

\section{References}

Albuquerque, L., Rainey, F. A., Chung, A. P., Sunna, A., Nobre, M. F., Grote, R., Antranikian, G. \& Da Costa, M. S. (2000). Alicyclobacillus hesperidum sp. nov. and a related genomic species from solfataric soils of São Miguel in the Azores. Int J Syst Evol Microbiol 50, 451-457.

Claus, D. \& Berkeley, R. C. W. (1986). Genus Bacillus Cohn 1872. In Bergey's Manual of Systematic Bacteriology, vol. 2, pp. 1105-1140. Edited by P. H. A. Sneath, N. S. Mair, M. E. Sharpe \& J. G. Holt. Baltimore: Williams \& Wilkins.

Deinhard, G., Blanz, P., Poralla, K. \& Altan, E. (1987a). Bacillus acidoterrestris sp. nov., a new thermotolerant acidophile isolated from different soils. Syst Appl Microbiol 10, 47-53.

Deinhard, G., Saar, J., Krischke, W. \& Poralla, K. (1987b). Bacillus cycloheptanicus sp. nov., a new thermoacidophile containing $\omega$-cycloheptane fatty acids. Syst Appl Microbiol 10, 68-73.

Dong, X. \& Cai, M. (2001). Determinative Manual for Routine Bacteriology (English translation). Beijing: Academic Press.

Dufresne, S., Bousquet, J., Boissinot, M. \& Guay, R. (1996). Sulfobacillus disulfidooxidans sp. nov., a new acidophilic, disulfideoxidizing, Gram-positive, spore-forming bacterium. Int $J$ Syst Bacteriol 46, 1056-1064

Gerhardt, P., Murray, R. G. E., Wood, W. A. \& Krieg, N. R. (editors) (1994). Methods for General and Molecular Bacteriology. Washington, DC: American Society for Microbiology.

Goto, K., Mochida, K. M., Asahara, M., Suzuki, M. \& Yokota, A. (2002). Application of the hypervariable region of the $16 \mathrm{~S} \mathrm{rDNA}$ sequence as an index for the rapid identification of species in the genus Alicyclobacillus. J Gen Appl Microbiol 48, 243-250.

Goto, K., Mochida, K., Asahara, M., Suzuki, M., Kasai, H. \& Yokota, K. (2003). Alicyclobacillus pomorum sp. nov., a novel thermo-acidophilic, endospore-forming bacterium that does not possess $\omega$-alicyclic fatty acids, and emended description of the genus Alicyclobacillus. Int J Syst Evol Microbiol 53, 1537-1544.

Goto, K., Mochida, K., Kato, Y., Asahara, M., Ozawa, C., Kasai, H. \& Yokota, A. (2006). Diversity of Alicyclobacillus isolated from fruit juices and their raw materials, and emended description of Alicyclobacillus acidocaldarius. Microbiol Cult Collect 22, 1-14.

Goto, K., Mochida, K., Kato, Y., Asahara, M., Fujita, R., An, S.-Y., Kasai, H. \& Yokota, A. (2007). Proposal of six species of moderately thermophilic, acidophilic, endospore-forming bacteria: Alicyclobacillus contaminans sp. nov., Alicyclobacillus fastidiosus sp. nov., Alicyclobacillus kakegawensis sp. nov., Alicyclobacillus macrosporangiidus sp. nov., Alicyclobacillus sacchari sp. nov. and Alicyclobacillus shizuokensis sp. nov. Int J Syst Evol Microbiol 57, 1276-1285.

Hippchen, B., Röll, A. \& Poralla, K. (1981). Occurrence in soil of thermo-acidophilic bacilli possessing $\omega$-cyclohexane fatty acids and hopanoids. Arch Microbiol 129, 53-55. 
Hiraishi, A., Inagaki, K., Tanimoto, Y., Iwasaki, M., Kishimoto, N. \& Tanaka, H. (1997). Phylogenetic characterization of a new thermoacidophilic bacterium isolated from hot springs in Japan. J Gen Appl Microbiol 43, 295-304.

Imperio, T., Viti, C. \& Marri, L. (2008). Alicyclobacillus pohliae sp. nov., a thermophilic, endospore-forming bacterium isolated from geothermal soil of the north-west slope of Mount Melbourne (Antarctica). Int J Syst Evol Microbiol 58, 221-225.

Jiang, C.-Y., Liu, Y., Liu, Y.-Y., You, X.-Y., Guo, X. \& Liu, S.-J. (2008). Alicyclobacillus ferrooxydans sp. nov., a ferrous-oxidizing bacterium from solfataric soil. Int J Syst Evol Microbiol 58, 2898-2903.

Johnson, D. B. \& Hallberg, K. B. (2007). Techniques for detecting and identifying acidophilic mineral-oxidizing microorganisms. In Biomining, pp. 237-262. Edited by D. E. Rawlings \& D. B. Johnson. Heidelberg: Springer-Verlag.

Karavaiko, G. I., Bogdanova, T. I., Tourova, T. P., Kondrat'eva, T. F., Tsaplina, I. A., Egorova, M. A., Krasil'nikova, E. N. \& Zakharchuk, L. M. (2005). Reclassification of 'Sulfobacillus thermosulfidooxidans subsp. thermotolerans' strain $\mathrm{K} 1$ as Alicyclobacillus tolerans sp. nov. and Sulfobacillus disulfidooxidans Dufresne et al. 1996 as Alicyclobacillus disulfidooxidans comb. nov., and emended description of the genus Alicyclobacillus. Int J Syst Evol Microbiol 55, 941-947.

Kimura, M. (1980). A simple method for estimating evolutionary rates of base substitutions through comparative studies of nucleotide sequences. J Mol Evol 16, 111-120.

Kovalenko, E. V. \& Malakhova, P. T. (1983). The spore-forming ironoxidizing bacterium Sulfobacillus thermosulfidooxidans. Mikrobiologiia 52, 962-966 (in Russian).

Kumar, S., Tamura, K. \& Nei, M. (2004). MEGA3: integrated software for molecular evolutionary genetics analysis and sequence alignment. Brief Bioinform 5, 150-163.
Marmur, J. \& Doty, P. (1962). Determination of the base composition of deoxyribonucleic acid from its thermal denaturation temperature. J Mol Biol 5, 109-118.

Nicolaus, B., Improta, R., Manca, M. C., Lama, L., Esposito, E. \& Gambacorta, A. (1998). Alicyclobacilli from an unexplored geothermal soil in Antarctica: Mount Rittmann. Polar Biol 19, 133-141.

Norris, P. R., Clark, D. A., Owen, J. P. \& Waterhouse, S. (1996). Characteristics of Sulfobacillus acidophilus sp. nov. and other moderately thermophilic mineral-sulphide-oxidizing bacteria. Microbiology 142, 775-783.

Saitou, N. \& Nei, M. (1987). The neighbor-joining method: a new method for reconstructing phylogenetic trees. Mol Biol Evol 4, 406-425.

Simbahan, J., Drijber, R. \& Blum, P. (2004). Alicyclobacillus vulcanalis sp. nov., a thermophilic, acidophilic bacterium isolated from Coso Hot Springs, California, USA. Int J Syst Evol Microbiol 54, 1703-1707.

Thompson, J. D., Gibson, T. J., Plewniak, F., Jeanmougin, F. \& Higgins, D. G. (1997). The CLUSTAL_X windows interface: flexible strategies for multiple sequence alignment aided by quality analysis tools. Nucleic Acids Res 25, 4876-4882.

Tsuruoka, N., Isono, Y., Shida, O., Hemmi, H., Nakayama, T. \& Nishino, T. (2003). Alicyclobacillus sendaiensis sp. nov., a novel acidophilic, slightly thermophilic species isolated from soil in Sendai, Japan. Int J Syst Evol Microbiol 53, 1081-1084.

Wakeman, K., Auvinen, H. \& Johnson, D. B. (2008). Microbiological and geochemical dynamics in simulated-heap leaching of a polymetallic sulfide ore. Biotechnol Bioeng, doi:10.1002/bit.21951

Wisotzkey, J. D., Jurtshuk, P., Jr, Fox, G. E., Deinhard, G. \& Poralla, K. (1992). Comparative sequence analyses on the $16 \mathrm{~S}$ rRNA (rDNA) of Bacillus acidocaldarius, Bacillus acidoterrestris, and Bacillus cycloheptanicus and proposal for creation of a new genus, Alicyclobacillus gen. nov. Int J Syst Bacteriol 42, 263-269. 